\title{
MONITOREO Y EVALUACIÓN DEL GRADO DE CONTAMINACIÓN EN LOS LABORATORIOS DE LA FACULTAD DE QUÍMICA E INGENIERÍA QUÍMICA (FQIQ) Y SU IMPACTO EN LA SALUD Y MEDIO AMBIENTE
}

\author{
Juana Sandívar ${ }^{1 *}$, Ana Osorio ${ }^{1}$, Laura Lengua ${ }^{1}$, Norma Salas ${ }^{1}$, Hilda \\ Carhuancho', María Lino', Manuel Bejar, ${ }^{1}$ José Pino², Pilar Pino², \\ Sheyla Chero, ${ }^{1}$ Wilver Auccahuasi ${ }^{3}$
}

\begin{abstract}
RESUMEN
Se evaluó la hipótesis planteada, donde la exposición mínima de 20 horas semanales a varios reactivos, soluciones y solventes durante las prácticas académicas, limpieza de almacén de reactivos, de docentes, trabajadores y alumnos de la FQIQ de la UNMSM ocasiona un incremento de biomarcadores de exposición y efecto temprano. La frecuencia de micronúcleos en la mucosa bucal fue analizada para comprobar el efecto citogenético y citotóxico en dos poblaciones: Grupo Problema y Grupo Control, en dos etapas. Por cada población se tomó tres grupos de un total de 85 individuos, docentes (edad media: 56,6 $\pm 6,48$ ), trabajadores no docentes (edad media: 48,5 $\pm 17,5$ ) y estudiantes (edad media: $25,14 \pm 4,99$ ). Se realizó el test de micronúcleo $(\mathrm{Mn})$ en células epiteliales de la mucosa bucal, comparando los valores obtenidos de daño genotóxico y citotóxico para cada individuo y en cada toma de muestra; en el análisis estadístico se aplicó ANOVA con un valor de significancia de $95 \%$ de confiabilidad. Se encontró daño a nivel celular en la población problema en ambas etapas de la toma de muestra, principalmente a nivel de núcleo (aberraciones nucleares). El daño más frecuente en ambas etapas fue la anomalía de célula binucleada $(26,70 \pm 16,44)$; seguido de células con cariólisis $(7,17 \pm 5,89)$, picnosis $(3,70 \pm 2,46)$ y "huevo roto" $(3,17 \pm 3,92)$. No se encontró relación con respecto a la edad de los grupos evaluados en ninguna de las dos tomas. En cuanto a la relación entre los tipos de estamentos universitarios, los trabajadores no docentes presentan mayor daño $(47,00 \pm 16,46)$, seguido de los docentes $(29,50 \pm 14,50)$ y los estudiantes $(17,00 \pm 10,00)$. En conclusión, existe daño citotóxico, genotóxico y apoptótico en la población problema, probablemente por la exposición crónica de sustancias químicas que dañan la integridad del ADN celular.
\end{abstract}

Palabras clave: Monitoreo, agentes contaminantes, extractor de aire, micronúcleo, toxicidad, genotoxicidad.

\section{MONITORING AND EVALUATION OF THE DEGREE OF CONTAMINATION IN CHEMISTRY AND CHEMISTRY ENGINEERING LABORATORIES (FQIQ) AND ITS IMPACT ON HEALTH AND THE ENVIRONMENT}

\footnotetext{
Facultad de Química e Ingeniería Química. Universidad Nacional Mayor de San Marcos (UNMSM) Lima, Perú.

2 Facultad de Ciencias Biológicas. UNMSM. Lima, Perú.

Facultad de Ingeniería de Sistemas. Universidad Particular San Martin de Porras. Lima, Perú.

1* jsandivarr@yahoo.es
} 


\begin{abstract}
We evaluated the hypothesis that an exposure of at least 20 hours per week with reagents during academic practice or research projects of teachers, workers and students of Chemistry and Chemistry Engineering Faculty of San Marcos University, caused an increase in biomarkers of exposure of early effect. The frequency of micronuclei in the oral mucosa was analyzed to check the cytogenetic effect in two populations: Problem Group and Control Group.

In each population was taken three groups totaling 85 individuals: teachers (mean age: 56,6 \pm 6,48 ), non-teaching staff (mean age: 48,5 $\pm 17,5$ ) and students (mean age 25,14 $\pm 4,99$ ). We used the micronucleus test $(\mathrm{Mn})$ in epithelial cells of the oral mucosa. When comparing the values obtained from DNA damage for each individual and for statistical analysis, ANOVA was applied with a significance value of $95 \%$ reliability. Damage was found at the cellular level of the population problem, mainly at nucleus (nuclear aberrations). The most common injury was binucleated cell $(26,70 \pm 16,44)$, followed by cells with karyolysis $(7,17 \pm 5,89)$, pyknosis $(3,70 \pm 2,46)$ and "broken egg" $(3,17 \pm 3,92)$. No relationship was found with respect to age groups evaluated. As for the relationship between the types of the workers, non-teaching employees have greater damage $(47.00 \pm 16.46)$, followed by teachers $(29,50 \pm 14,50)$ and students $(17,00 \pm 10,00)$. In conclusion, there is cytotoxic, genotoxic and apoptotic damage in the population issue, probably by chronic exposure to chemicals that damage cellular DNA integrity.
\end{abstract}

Key words: Monitoring, pollutants agents, air extractor, micronucleus, toxicity, genotoxicity.

\title{
INTRODUCCIÓN
}

La contaminación ambiental es parte inherente en las actividades del hombre. El ser humano es un excelente agente de cambio en el planeta y no escapa a los efectos de la destrucción que él mismo provoca, introduciendo al ambiente decenas de miles de compuestos químicos ${ }^{1,2,3}$, agregando cada año al mercado nuevos productos y subproductos; la mayoría de los cuales no han sido evaluados responsablemente respecto a su toxicidad y posible impacto sobre la población y el ambiente ${ }^{4,5,6}$.

Esos cambios son el producto de situaciones tales como el aumento de enfermedades oncológicas $^{7}$ y emergentes, la eliminación selectiva de especies y la alteración de los hábitats. Es imperiosa la necesidad de evaluar y detectar el impacto de las actividades antrópicas sobre los organismos y su ecosistema. Una alternativa es considerar el uso de bioindicadores ${ }^{8,9,10}$, los cuales proveen la posibilidad de obtener información sobre las moléculas contaminantes, directamente sobre sus receptores biológicos; una forma complementaria es cuantificar el grado de contaminación del aire mediante el uso de sensores de tipo electroquímico.

\section{PARTE EXPERIMENTAL}

Los individuos del grupo problema son profesores, trabajadores y alumnos de la Facultad de Química e Ingeniería Química que, debido a su exposición ocupacional o académica, se encuentran a diario en contacto con distintos tipos de reactivos, solventes, gases, etc., mientras que los individuos del grupo control son alumnos, docentes y trabajadores de la Facultad de Letras y Ciencias Sociales, que no están expuestos a productos químicos en su labor ocupacional o académica.

Se realizó un descarte de individuos fumadores y/o individuos que estaban o habían dejado de consumir antibióticos, antidepresivos, etc., en un mínimo de tres meses previos al inicio de la parte experimental, para descartar, así, cualquier factor externo que nos diese un falso positivo, fuera de la exposición a las sustancias tóxicas, esto se realizó mediante una encuesta. A los 
individuos que pertenecen al grupo problema se les realizó una capacitación de buenas prácticas de laboratorios de Química; en el uso y manejo de reactivos, durante la cual se tomaron temas sobre bioseguridad y mantenimiento adecuado del instrumental, se realizó una toma de muestra previa a la capacitación y luego de seis meses se realizó otra.

El instrumento metodológico más validado y desarrollado para evaluar el daño ocasionado por cualquier mutagénico, como la exposición continua a estos distintos tipos de reactivos, es el Test de Micronúcleos ( $\mathrm{Mn}$ ) en células de la mucosa bucal, que consiste en la determinación de alteraciones nucleares, detectando lesiones a nivel citogenético ${ }^{11,12}$.

\section{Test de Micronúcleo:}

Se contó y evaluó 2000 células mononucleadas por individuo, siguiendo los criterios de evaluación de Mn sugeridos por Titenko-Holland et al. ${ }^{13}$. Las muestras epiteliales de descamación de la mucosa bucal se obtuvieron de los usuarios de los laboratorios de Química e Ingeniería Química; se realizó un hisopado friccionando ligeramente el interior de las mejillas de cada individuo con un hisopo, sin tocar los dientes ni la lengua. Se preparó como mínimo dos láminas portaobjetos por individuo, lo que facilitó la obtención de un gran número de células al ser analizadas; la tinción se realizó con el colorante May Grunwald - Giemsa (Sigma) y previamente al recuento, los códigos de las láminas se cubrieron para evitar sesgos en los resultados.

- Con un hisopo estéril se procedió a realizar un raspado de la cara interna de las dos mejillas, evitando el contacto del hisopo con otras partes de la boca.

- Se sumergió el hisopo inmediatamente en un tubo Eppendorf de 1,5 ml con 1,0 ml de buffer Tris-EDTA.

- Se centrifugó por 10 minutos a 1500 rpm; se descartó el sobrenadante y se añadió 1,0 ml delbufferanterior y seresuspendióel botón conuna micropipetade 1000ìl.

- Luego de los lavados, se descartó el sobrenadante dejando una cantidad suficiente de tampón para tener una buena concentración de células.

- Se realizó un frotis con $20 \mu \mathrm{l}$ de muestra por gota en cada porta objeto y se dejó que secara a temperatura ambiente por 30 minutos. Seguidamente se fijó con metanol absoluto frío durante 15 minutos.

- $\quad$ Finalmente se coloreó con Giemsa al $2 \%$ por 15 minutos.

- Se realizó un recuento de 2000 células por individuo, con el microscopio de campo claro con un aumento del $1000 \mathrm{X}$.

\section{Análisis estadístico}

Para el análisis de los resultados se realizó un estudio descriptivo y analítico utilizando el paquete estadístico SPSS 11.5. Los datos obtenidos en el estudio fueron procesados dependiendo de los resultados; primero se comprobó si tienen una distribución normal con el test de Kolmogorov-Smirnov; luego del análisis, para verificar la normalidad, se procedió con los Test de Bonferroni y Tukey, respectivamente, para comparar la frecuencia de Micronúcleos y Aberraciones Nucleares presentes en las células de descamación bucal. Con un nivel de significancia $(\mathrm{p}<0,05)$.

El presente estudio contó con tres poblaciones: docente, no docentes y estudiantes que hacen uso de los laboratorios de la FQIQ y docentes, no docentes y alumnos que son ajenos a los laboratorios (Facultad de Letras).

En el análisis estadístico se empleó las técnicas para analizar los datos como: desviación estándar, coeficiente de variación, histograma y prueba no paramétricas de t-student).

\section{Detección de gases $\mathrm{SO}_{2}$ y $\mathrm{NO}_{2}$}

En un primer momento para generar el $\mathrm{SO}_{2}$ se obtuvo oxígeno por desplazamiento de agua, 
para ello se calentó una mezcla de $\mathrm{KClO}_{3}$ y $\mathrm{MnO}_{2}$ en tubo de ensayo seco y con tapón provisto de una salida lateral. El oxígeno se recogió en un matraz Erlenmeyer y se tapó herméticamente. A continuación, en una cuchara de combustión se calentó azufre rómbico en polvo hasta que se encendiera para luego introducirlo en el matraz con oxígeno. Una vez que la llama está casi apagada, se retira la cuchara de combustión y se tapa; en ese momento se forma el $\mathrm{SO}_{2}$ y algo de $\mathrm{SO}_{3}$. Posteriormente, se destapa el matraz y se deja que el $\mathrm{SO}_{2}$ escape al ambiente. Es en ese momento que el analizador de gases Q-RAE modelo PGM-7800 empieza a captar a este gas por espacio de dos horas.

Para generar el $\mathrm{NO}_{2}$ se colocó láminas de cobre en un matraz quitasato y luego se agregó desde una pera de decantación $\mathrm{HNO}_{3(\mathrm{c})}$, el cual se dejó gotear. En un tubo de ensayo se recogió el $\mathrm{NO}_{2}$ e inmediatamente se expuso al medio ambiente para que a su vez el analizador de gases QRAE modelo PGM-7800 detecte la presencia de este gas por espacio de dos horas.

\section{RESULTADOS Y DISCUSIÓN}

Las personas expuestas por más de 20 horas semanales a los diferentes solventes, soluciones, metales, etc. que se encuentran en los diversos laboratorios de la Facultad de Química e Ingeniería Química de la UNMSM fueron los integrantes del Grupo Problema (GP). Se encuentran alumnos, docentes y trabajadores, y sus edades oscilan entre 21 - 74 años. Los individuos integrantes del Grupo Control (GC) son alumnos, docentes y trabajadores no docentes de las Escuelas Profesionales de Arte y de Restauración de la Facultad de Letras.

Las características generales de los individuos de ambos grupos son aquellos que no han consumido antibióticos, antidepresivos, analgésicos, etc., que pudieran influir en las diversas anomalías nucleares que se estudiaron; y que tampoco hayan consumido tabaco o cigarro. En cuanto al hábito de beber alcohol, Castillo-Cadena et al. ${ }^{14}$ consideraron como criterio el consumo diario de alcohol y ninguna persona bebe de esa manera, por lo que no fueron considerados bebedores consuetudinarios. Tampoco deben haber estado expuestos en el último año a rayos $\mathrm{X}$.

Luego de la evaluación de 2000 células de la mucosa bucal por individuo por etapa de toma de muestra en la población control $(n=35)$ y la población problema $(n=37)$, los resultados obtenidos entre el grupo control y el grupo problema en las distintas aberraciones nucleares como: células binucleadas, células en cariolisis, células en picnosis y células en huevo roto, fueron significativas para todas las aberraciones nucleares estudiadas $\mathrm{p}<0,001$ (test student) en la primera etapa, se encontró una media mayor de células con núcleo binucleado $(3,0050 \pm$ $0,4989)$, seguido de células con núcleo en picnosis $(0,8166 \pm 0,1796)$, células con cariolisis $(0,4416 \pm 0,0743)$, y células con micronúcleo sin separación (broken egg) $(0,2583 \pm 0,1041)$ (tabla 1) y (figura 1).

Tabla 1. Comparación entre el grupo control y grupo problema de las anormalidades citotóxicas encontradas antes de la capacitación.

\begin{tabular}{ccccc}
\hline \multirow{2}{*}{$\begin{array}{c}\text { Grupo control vs Grupo problema 1ra. toma } \\
\text { label }\end{array}$} & binucleada & broken egg & picnosis & cariolisis \\
Grupo control & $0,1280 \pm 0,3189$ & $0,0000 \pm 0,0000$ & $0,0000 \pm 0,0000$ & $0,0120 \pm 0,0067$ \\
Grupo & $3,0050 \pm 0,4989$ & $0,2583 \pm$ & $0,8167 \pm$ & $0,4417 \pm$ \\
problema & $*$ & $0,1041^{*}$ & $0,1796^{*}$ & $0,0743^{*}$ \\
\hline
\end{tabular}

* SPSS versión $17, \mathrm{t}$ student,p $<0,05$ 


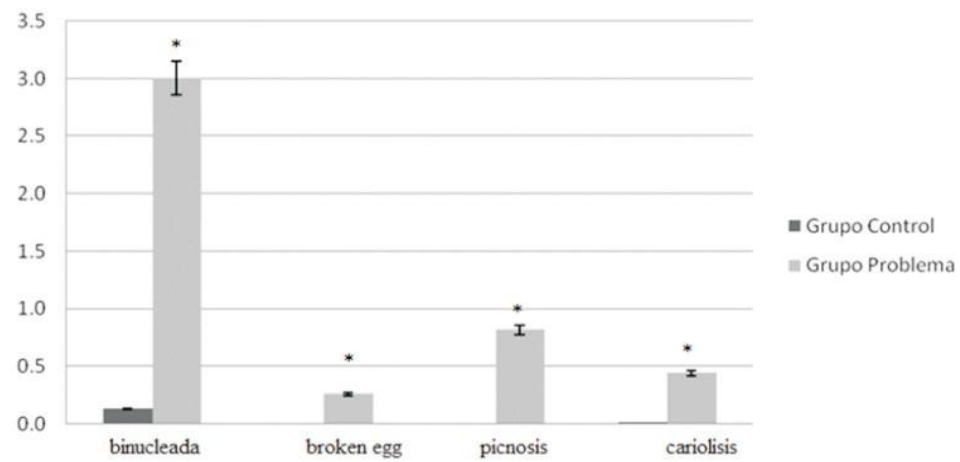

Figura 1. Comparación entre el grupo control y grupo problema de las anormalidades citotóxicas encontradas antes de la capacitación. * SPSS versión 17, t student,p $<0,05$

Los resultados obtenidos de la toma de muestra entre el grupo problema y el grupo control después de la capacitación, en las distintas aberraciones nucleares como: células binucleadas, células en cariolisis, células en picnosis y células en huevo roto, fueron significativas para todas las aberraciones nucleares estudiadas $\mathrm{p}<0,001$ (test student) en la segunda etapa, se encontró una media mayor de células con núcleo binucleado $(3,0462 \pm 1,2610)$, seguido de células con núcleo en picnosis $(0,7538 \pm 0,7824)$, células con cariolisis $(0,4077 \pm 0,5143)$ y células con micronúcleo sin separación (broken egg) $(0,2385 \pm 0,5819)$ (tabla 2) y (figura2).

Tabla 2. Comparación entre el grupo control y grupo problema de las anormalidades citotóxicas encontradas después de la capacitación.

\begin{tabular}{ccccc}
\hline & \multicolumn{4}{c}{ Grupo control vs Grupo problema 2da. toma } \\
binucleada & broken egg & picnosis & cariolisis \\
Grupo problema & $3,0462 \pm 1,2610^{*}$ & $0,2385 \pm 0,5819^{*}$ & $0,7538 \pm 0,7824^{*}$ & $0,4077 \pm 0,5143^{*}$ \\
Grupo control & $0,1280 \pm 0,3953$ & $0,0000 \pm 0,0000$ & $0,0000 \pm 0,0000$ & $0,120,5701$ \\
\hline
\end{tabular}

* SPSS versión $17, \mathrm{t}$ student,p $<0,05$

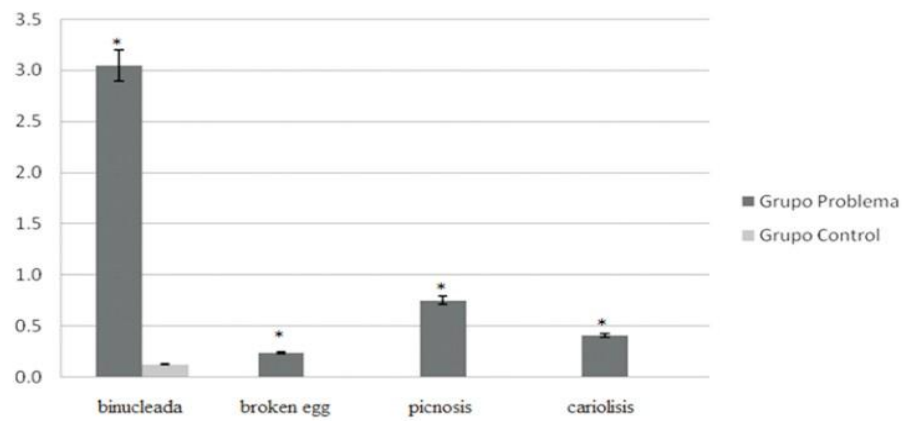

Figura 2. Comparación entre el grupo control y grupo problema de las anormalidades citotóxicas encontradas después de la capacitación. * SPSS versión 17, t student,p <0,05 
Los resultados obtenidos entre el grupo problema de las dos tomas de muestras, antes y después de la capacitación en las distintas aberraciones nucleares como células binucleadas, células en cariolisis, células en picnosis y células en huevo roto, no fueron significativas en las aberraciones nucleares estudiadas p<0,001 (test student) (tabla 3) y (figura 3).

Tabla 3. Comparación en el Grupo problema entre las dos tomas de muestras. GP 1. Grupo problema primera toma, GP 2. Grupo problema segunda toma.

\begin{tabular}{ccccc}
\hline & binucleada & broken egg & picnosis & cariolisis \\
GP 1 & $3,0050 \pm 0,4989$ & $0,2583 \pm 0,1041$ & $0,8167 \pm 0,1796$ & $0,4417 \pm 0,0743$ \\
GP 2 & $3,0462 \pm 1,2610$ & $0,2385 \pm 0,5819$ & $0,7538 \pm 0,7824$ & $0,4077 \pm 0,5143$ \\
\hline
\end{tabular}

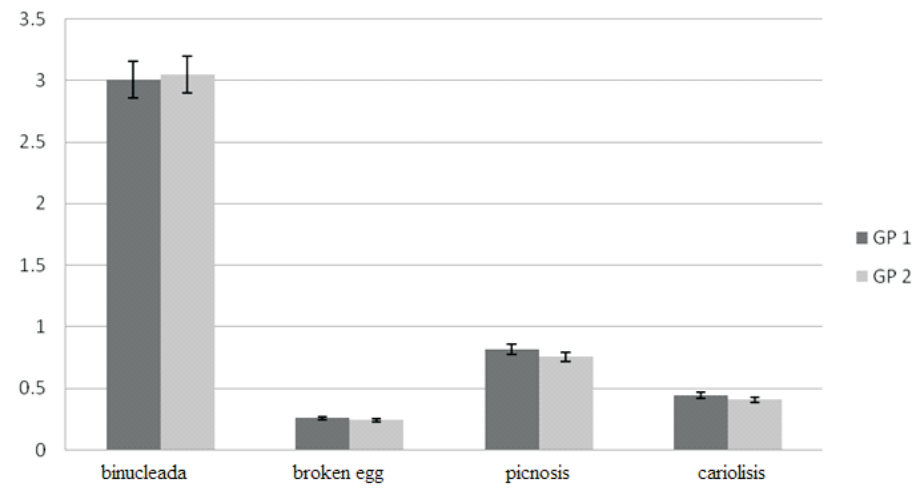

Figura 3. Comparación en el Grupo Problema entre las dos tomas de muestras. GP 1. Grupo Problema primera toma, GP 2. Grupo Problema segunda toma.

Según lo establecido por otros estudios ${ }^{15}$, el promedio normal de las frecuencias de micronúcleos y anomalías nucleares varía considerablemente de una población a otra $(0,03 \%$ a $0,47 \%$ ). Estas diferencias dependen de factores genéticos y ambientales tales como la alimentación, la edad y el sexo ${ }^{16}$.

Los promedios de las frecuencias de las anomalías nucleares encontradas antes y después de la capacitación sugieren que no ha habido ninguna mejora en la calidad de vida de los individuos que pasan más de 20 horas semanales en los laboratorios de algunos departamentos de Química e Ingeniería Química de la UNMSM; la exposición continua a los diferentes solventes, soluciones, metales que están en los diversos laboratorios de la institución hacen un daño significativo en el material genético. La prueba t-Student aplicada para analizar los valores promedio de las frecuencias de aberraciones nucleares de las muestras de las expuestas con respecto a las no expuestas, determinó que existen diferencias significativas entre el grupo problema y el grupo control, en ambas tomas de muestra.

Estudios en soldadores de la ciudad de Cartagena (Colombia) demuestran que la exposición continúa a ciertos metales como cadmio, plomo, arsénico, mercurio, zinc, cobre y aluminio, 
en conjunto o por separado, pueden ocasionar desórdenes gastrointestinales, diarreas, estomatitis, temblores, hemoglobinuria, ataxia, parálisis, vómitos y convulsiones, depresión y neumonía, cuando se inhalan vapores volátiles y humos. La naturaleza de los efectos puede ser tóxica (aguda, crónica o subcrónica), neurotóxica, cancerígena, mutagenética o teratogenética $^{7,11}$.

El tejido de los carrillos de la boca es un epitelio escamoso estratificado no queratinizado muy grueso que protege a los tejidos profundos de la fuerza mecánica de la masticación y forma una barrera poco permeable $e^{10,11}$. Las células de las capas superficiales (que corresponden a la muestra analizada) se exponen fácilmente a cualquier sustancia tóxica; sin embargo, la saliva presente en toda la mucosa de la boca protege de sustancias poco solubles en agua, como es el caso de muchos metales y reactivos ${ }^{6,7}$, por lo que se debería enriquecer el estudio con otras pruebas que determinen mayores resultados que lleven a cambios favorables en la seguridad de la población estudiada.

Los estudios de tipo epidemiológico ${ }^{8,9,10}$ han señalado a muchas de estas sustancias como potencialmente carcinógenas, neurotóxicas, teratógenas, mutagénicas, fetotóxicas y de causar efectos negativos en el sistema reproductor de ciertos animales ${ }^{11}$, por lo tanto es necesario continuar una estrecha vigilancia sobre la salud de los alumnos, docentes y trabajadores.

Al agrupar las anormalidades nucleares en tres diferentes categorías: genotoxicidad, citotoxicidad y apoptosis, se observa que las dos últimas son significativamente más elevadas en la población problema. La genotoxicidad es un proceso que puede llevar a apoptosis (muerte celular programada) y a un aumento en la frecuencia de células con núcleos en cariolisis y en cariorresis puede significar genotoxicidad; por lo tanto, los individuos del grupo problema están más propensos a presentar problemas de salud.

El uso continuo de biomarcadores por el personal que trabaja en laboratorios se expresaría en una mejora en la seguridad de cada integrante al emplear equipos especializados para la protección o la detección de exposición mínima de estos contaminantes, lo que llevaría a una mejora en la calidad de vida y disminución del deterioro de la salud.

Los biomarcadores son herramientas de monitoreo de detección de exposiciones de sustancias agresivas que afectan a la población en general, dependiendo de la ocupación que realiza, favorecería principalmente a las personas que están expuestas continuamente a varios tipos de contaminantes, como solventes, metales pesados, relaves mineros, plaguicidas, entre otros.

\section{CONCLUSIONES}

Según los resultados del test de micronúcleos (Mn) del estudio en células de la mucosa bucal del personal analizado para comprobar el efecto citogenético y citotóxico, se puede concluir que sí existe diferencia significativa entre los individuos del grupo problema frente a los individuos del grupo control, por lo que se puede afirmar que sí existe daño citotóxico, genotóxico y apoptótico en la población problema, probablemente por la exposición crónica de sustancias químicas que dañan la integridad del ADN celular.

Que de la población problema más perjudicada por el efecto citogenético y citotóxico corresponde a los trabajadores seguido de los docentes y alumnos en el orden que se indica, probablemente porque ellos tienen mayor tiempo de exposición en los ambientes y el desconocimiento de la toxicidad de ciertos reactivos químicos.

Las anormalidades citotóxicas corresponden en mayor porcentaje a la presencia de células binucleadas seguidas de la picnosis, encontrándose menor efecto en la cariolisis y broken eggs.

Se ha encontrado valores altos de células binucleadas en el rango de 47,00 $\pm 16,46$.

Para una mejor calidad de vida de los usuarios de laboratorios: estudiantes, docentes y no 
docentes y revertir el daño que se ha ocasionado se debe tomar en cuenta las siguientes sugerencias:

Se debe considerar tomar medidas de seguridad en el uso, manejo y almacenaje de los diversos reactivos y materiales que se procesan en la Facultad de Química e Ingeniería Química, sobre todo tomando en cuenta la fecha de caducidad de los reactivos y su efecto negativo en la calidad de vida de alumnos, docentes y trabajadores.

Proponer que los laboratorios cuenten con ductos de ventilación para que en las noches se expulse los gases acumulados.

Se debe hacer convenios con empresas para propiciar charlas de bioseguridad e higiene industrial.

Establecer una programación de mantenimiento permanente de las campanas extractoras de todos los laboratorios.

La FQIQ debe hacer un contrato con empresas que eliminen residuos químicos potencialmente peligrosos semestralmente.

Que el área competente gestione la contratación de una empresa para que tome las muestras de los gases contaminantes en el Laboratorio de Química Inorgánica, ubicada en el segundo piso, en las fechas que corresponda a la cuarta, quinta y sexta semana del segundo semestre académico dado que en este periodo se trabaja con azufre y nitrógeno. Esta propuesta puede ser ampliada a todos los otros laboratorios de la FQIQ y así evitar que los límites estén superando lo permisible.

\section{AGRADECIMIENTO}

Los integrantes de la presente investigación agradecemos a los Doctores Marco Martos y Nelson Tapia, Decanos de las Facultades de Letras y Química e Ingeniería Química, respectivamente, por haber brindado las facilidades en el desarrollo del trabajo. Así también el agradecimiento a los estudiantes, docentes y trabajadores no docentes de las Escuelas Profesionales de Arte y Restauración, quienes fueron nuestra población control de la Facultad de Letras y también a los estudiantes, docentes y trabajadores no docentes, quienes fueron nuestra población problema de la FQIQ, ambas de la UNMSM.

\section{BIBLIOGRAFÍA}

1. Bernard, A. Renal and Neurological Effects Heavy Metals in the Environment. Encyclopedia of Environmental Health. 2011, 801-805.

2. Ashby, J., W. Anwar, W.W. Au, A. Massoud \& J. M. Gentile. Genetic toxicology in developing countries: comments and recommendations. Environ. Health Perspect. Suppl. 1993; 101(3): 335-338.

3. Turkez, H., Geyikoglu, F., Tatar, A., Keles, MS., Kaplan,I. The effects of some boron compounds against heavy metal toxicity in human blood. Experimental and Toxicologic Pathology. 2010; 64(1-2):93-101.

4. Hayes, W.J. Pesticide problems and their solutions. In: Hayes, W. J. and E.R. Laws (eds). Handbook of pesticide toxicology. Vol 1. General principles. Academic, San Diego. 1991.pp. 27-29.

5. Edwards, R., D.H. Ferry \& W.A. Temple. Fungicides and related compounds, 1991.pp. 1409-1470.

6. Hayes, W. J. \& E. R. Laws (eds.). Handbook of pesticide toxicology. Vol. 3. Classes of pesticides. Academic, San Diego. pp. 463-496. 
7. Pisani, P. Burden of cancer in developing countries. In: N. Pearce, E. Matos, H. Vainio., P. Boffetta \& M. Kogevinas (eds.). Occupational cancer in developing countries. IARC. Scientific Publications. No. 129. Lyon. 1994. pp. 31-39.

8. Lohman, P.H. M., J. D.Jansen \& R. A. Baan. Comparison of various methodologies with respect to specificity and sensitivity in biomonitoring occupational exposure to mutagens and carcinogens. 1984. pp. 259-277.

9. Berlin A., M. Draper, K. Hemminki \& H. Vainio. (eds.). Monitoring human exposure to carcinogenic and mutagenic agents. IARC. Scientific Publications. No. 59. Lyon. Francia. 457 pp.

10. Martins, RA., da Silva, G., Aguilar, O., Ribeiro, RA. Biomonitoring of oral epithelial cells in petrol station attendants: Comparison between buccal mucosa and lateral border of the tongue. Environment International. 2009; 35:1062-1065.

11. Tolbert, P.E., C.M. Shy \& J.W.Allen. Micronuclei and other nuclear anomalies in buccal smears a field test in snuff users. Am. J. Epidemiol. 1991; 134: 840-850

12. Wesseling, C., A. Ahlbom, D. Antich, A. Rodriguez \& R. Castro. Cancer in banana plantation workers in Costa Rica. Int. J. Epid. Assoc. 1996. 25: 1125-1131.

13. Titenko-Holland, N., L.E. Moore \& M.T. Smith. Measurement and characterization of micronuclei en exfoliated human cells by fluorescence in situ hybridization with a centromeric probe. Mut. Res. 1994. 312:39-50.

14. Castillo-Cadena J., Tenorio-Vieyra L., Quintana-Carabia A., García-Fabila M., RamírezSan Juan E. y Madrigal-Bujaidar E. Determination of DNA damage in floriculturist exposed to mixtures of pesticides. J. Biomed. Biotechnol. 2006; (2): 1-12.

15. Pastor, S., A. Creus, N. Xamena, C. Siffel \& R. Marcos. Occupational exposure to pesticidas and cytogenetic damage: Results of a hungarian population study using the micronucleus assay in lymphocytes and bucal cells. Environ. Mol. Mutagen. 2002; 40: 101-109.

16. Berne, R. \& M. Levi. Principles of physiology. Mosby/ Doyma. Barcelona, 1992, 680 p. 